\title{
Quantum Entanglement of Magnons Confined in Multilay- ered Co/Pd Ferromagnets
}

\section{Chidubem A. Nwokoye*, Abid Siddique, Lawrence H. Bennett and Edward Della Torre}

Department of Electrical and Computer Engineering, Institute for Magnetics Research, The George Washington University, Washington, USA

\begin{abstract}
Quantum entanglement is a unique physical phenomenon that arises from the correlation between two or more quantum systems. The fundamental aspects of magnon entanglement have been theoretically studied. Developing technologies that exploits quantum entanglement will have enormous applications for sensors and quantum computing. In this paper, we discuss the results of an experimental study of magnon entanglement in multilayered Co/Pd ferromagnets. We observed an entanglement between the magnons in two spatially separated $\mathrm{Co} / \mathrm{Pd}$ ferromagnets. Our findings will aid in developing novel magnonic devices.
\end{abstract}

\section{Keywords}

Ferromagnet, Entanglement, Hysteresis, Magneto-optical kerr effect, Bose-Einstein condensation (BEC)

\section{Introduction}

Quantum entanglement is one of the most unique phenomena in modern physics and is a resource to many potential applications such as quantum teleportation, cryptography, and quantum communications. It shows the strong correlation between the systems of particles which cannot be described by classical mechanics. In the 1930s, Einstein, et al. [1] concluded that the quantum-mechanical description of a physical reality given by wave functions is not complete but believed that the theory is possible. Over the past few decades, significant research has been made on a variety of systems like atomic ensembles [2-5] and ion-traps [6] to demonstrate the photon-photon entanglement [7] and spin-photon entanglement [8-10] of different quantum states. The spin-photon entanglement is demonstrated in semiconducting nanostructures like Quantum Dots (QDs) with single spins interacting with incoming photons. The Kerr or Faraday rotation of the polarization of photons is observed in [11] and it created a renewed interest in quantum magneto-optics by investigating the magnon-photon interaction and entanglement in metallic nanostructures.

A magneto-optical Kerr effect (MOKE) magnetometer has been previously used for the characterization of the magneto-optical properties of magnetic materials [12] at room and low temperatures but has not been utilized for the exploration of entanglement of magnons. Theoretically the fundamental aspects of entanglement of magnons were presented in [13]. We here experimentally report the magnon entanglement in multi-layer $\mathrm{Co} /$ $\mathrm{Pd}$ thin films at low temperatures which are spatially separated inside a cryostat in a MOKE magnetometer.

\section{Experiment}

The Co/Pd test sample was grown by molecular beam epitaxy on a silicon dioxide substrate using electron beam evaporation. We followed a similar fabrication procedure illustrated in the work by Nwokoye, et al. [14]. The test sample composition is: $22 \mathrm{~nm} \mathrm{Si}<100>/ \mathrm{SiO}_{2}$ substrate,

*Corresponding author: Chidubem A Nwokoye, Carderock Division, Naval Surface Warfare Center, West Bethesda, Maryland 20817, USA, E-mail: dubem@gwmail.gwu.edu

Received: May 18, 2017: Accepted: August 04, 2017: Published: August 31, 2017

Copyright: (C) 2017 Nwokoye CA, et al. This is an open-access article distributed under the terms of the Creative Commons Attribution License, which permits unrestricted use, distribution, and reproduction in any medium, provided the original author and source are credited. 
$10 \mathrm{~nm}$ of Pd, ( $0.3 \mathrm{~nm}$ of Co and $1 \mathrm{~nm}$ of Pd) $15,10 \mathrm{~nm}$ of $\mathrm{Pd}$ and $1.5 \mathrm{~nm}$ of Fe. The sample was designed to exhibit a high perpendicular magnetic anisotropy. The sample

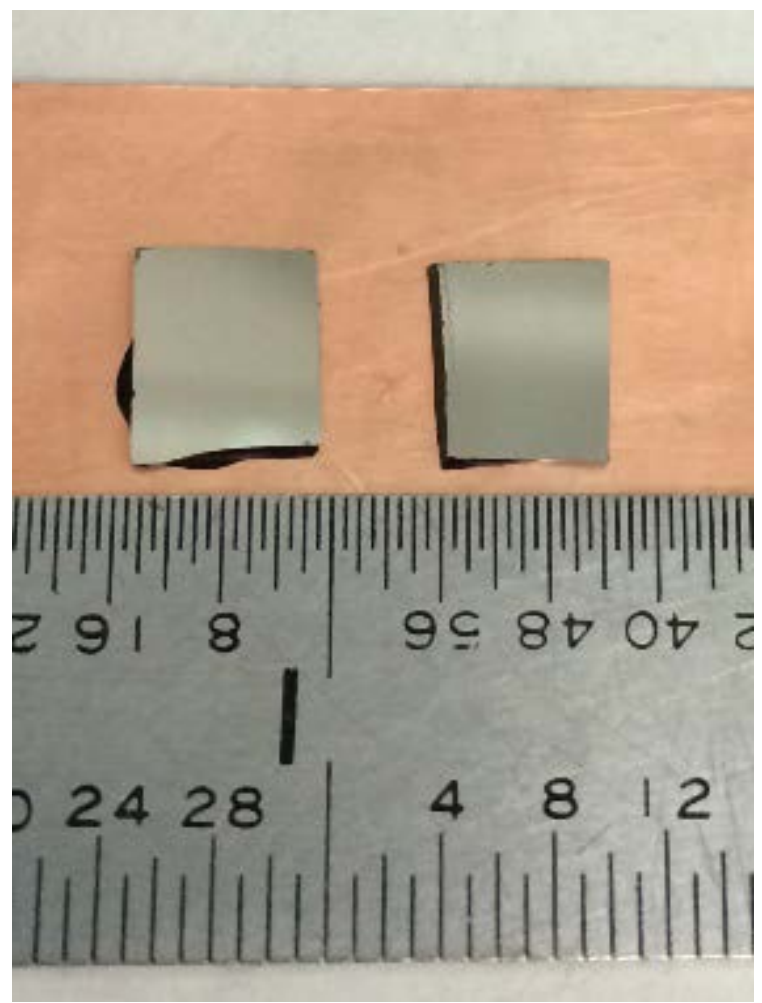

Figure 1: Picture of two Co/Pd samples cut from same sample and placed $3.5 \mathrm{~mm}$ apart, edge to edge. was cut into two pieces as shown in Figure 1 which were separated and glued on a thin rectangular copper sheet by a thermally conductive adhesive (Stycast $2850 \mathrm{FT} / 24$ $\mathrm{VL}$ ) capable of withstanding low temperatures down to 9 $\mathrm{K}$ and vacuum pressures lower than $1 \times 10^{-5}$ torr.

The copper sheet with the two samples (S1, S2) was placed inside a vacuum cryostat of an automated cryogen-free low-temperature PEM-based MOKE system. Details of the experimental setup and procedures are described in [12]. The block diagram of the setup is depicted in Figure 2. The design is used to study the correlation between magnons confined in two separated samples. The setup consists of two $638 \mathrm{~nm}$ red lasers (L1 and L2), Linear Polarizer (P1), PEM Modulator (P2), Optical CCR Cryostat (V), Electromagnet (E), Analyzer (A), Chopper (C), three Mirrors (M1, M2 and M3), and Laser Diode Detector (D).

(1) At room temperature, polar Kerr rotation hysteresis measurement on the sample S1 was recorded for applied magnetic fields ranging from $-4 \mathrm{kOe}$ to +4 kOe with L2 switched off. On the same sample S1, polar Kerr rotation aftereffect measurement with holding field at the coercivity field of approximately 970 Oe for 600 seconds was recorded. This is needed so that we could decisively identify the effect of the modulated laser from L2 on sample S1. Afterwards, L2 was switched on (allows modulated laser beam to be incident on sample S2) and both the polar Kerr rotation hysteresis and aftereffect

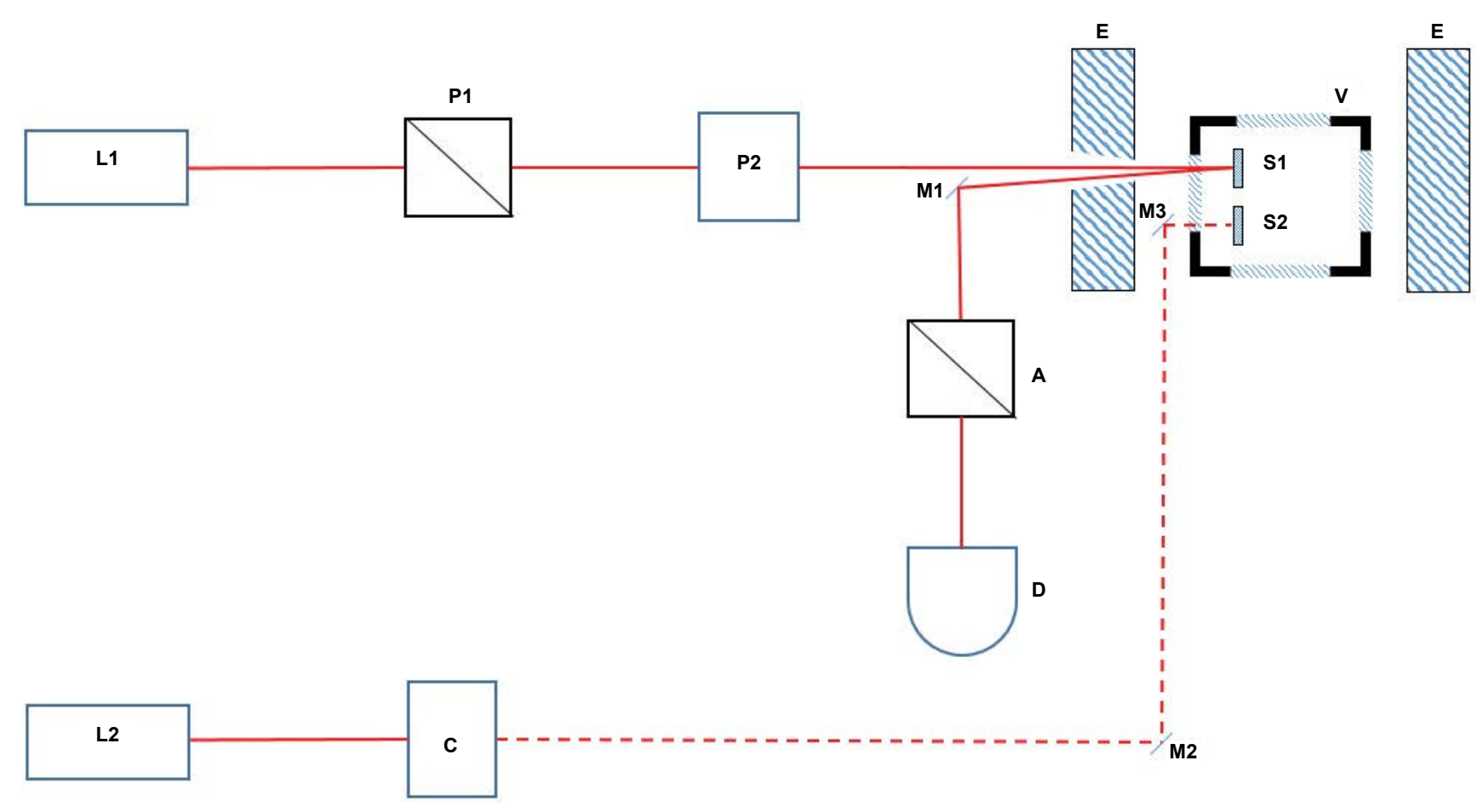

Figure 2: Block diagram of experimental setup. L1 \& L2 are laser sources, $P 1$ is a linear polarizer, P2 is a PhotoElastic Modulator (PEM) crystal, $E$ is an electromagnet, M1-3 are mirrors, $A$ is an analyzer, $V$ is vacuum cryostat, $C$ is variable frequency chopper, and $\mathrm{D}$ is a laser diode detector. 


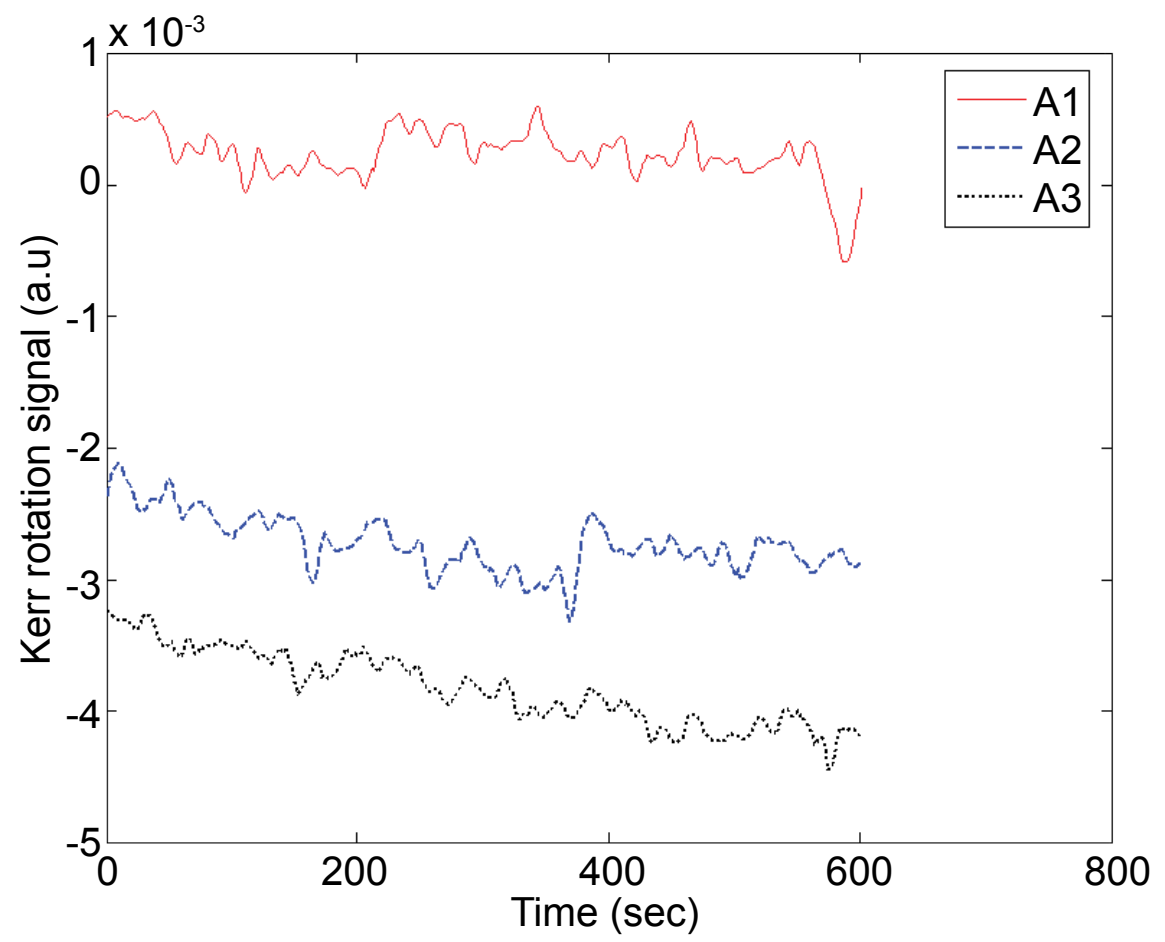

Figure 3: Kerr rotation signal time responses at coercivity field $(\approx 970 \mathrm{Oe})$. A1 represents measurement without external modulated laser (baseline). A2 represents measurement with external laser with modulation frequency of $1 \mathrm{kHz}$. A3 represents measurement with external laser with modulation frequency of $2 \mathrm{kHz}$.

measurements were repeated on sample S1 for various chopper modulation frequencies ranging from $0 \mathrm{~Hz}$ to $2 \mathrm{kHz}$. These measurements were performed in order to observe the coupling of the photon-magnon interaction in sample S2 onto sample S1 at room temperature.

(2) Keeping the temperature unchanged, the mirror M3 in Figure 2 was adjusted to redirect the modulated laser beam onto sample S1 in order to directly measure the influence of the modulated laser beam on the Kerr rotation of sample $\mathrm{S} 1$. Both the aftereffect and hysteresis Kerr rotation measurements were recorded.

(3) Following the procedure described in [12], low temperature measurements of the Kerr rotation hysteresis were recorded on the sample $\mathrm{S} 1$ at $9.5 \mathrm{~K}$ and $60 \mathrm{~K}$ with the following scenarios: (i) Laser L2 switched off; (ii) Laser L2 switched on and modulated laser beam incident on sample S2; (iii) Laser L2 switched on and modulated laser beam incident on sample $\mathrm{S} 1$. The proceeding paragraphs discuss the results of the performed experiments.

\section{Results and Discussions}

The Kerr rotation aftereffect measurements at room temperature and holding field of 970 Oe for scenarios A1-3 are shown in Figure 3, where A1 represents measurement with L2 switched off and there was no modulated laser on sample S1. A2 represents measurement with L2 switched on and mirror M3 is adjusted to allow the modulated laser with modulation frequency of $1 \mathrm{kHz}$ to be incident on sample S1. A3 represents measurement with L2 switched on and mirror M3 is adjusted to allow the modulated laser with modulation frequency of $2 \mathrm{kHz}$ to be incident on sample S1. We find from Figure 3 that the frequency of the incident modulated laser introduces amplitude offset to the Kerr rotation signal. This offset is evident in the aftereffect measurements because the aftereffect records the Kerr rotation signal over a time span. The hysteresis major loops measurement at room temperature are shown in Figure 4 and the curves depict no difference at the coercive field but a small difference at the remanence (zero applied field) and saturation field.

The result of the low temperature measurements at $60 \mathrm{~K}$ are depicted in Figure 5. As shown, D1 depicts the Kerr rotation of sample S1 when laser L2 is switched on and modulated laser beam is incident on sample S2 at 60 $\mathrm{K}$. The D1 curve measures the amount of coupling from sample S2 onto sample S1. D2 depicts the Kerr rotation of sample S1 when laser L2 is switched on and the modulated laser beam is incident on sample S1 at $60 \mathrm{~K}$. D2 measures the influence of modulated laser beam from L2 on Sample S1. D3 depicts the Kerr rotation of sample $\mathrm{S} 1$ when laser L2 is switched off at $60 \mathrm{~K}$. The D3 curve contains no influence of the external laser beam from L2 on sample S1. We noticed the following: (i) The Kerr rotation hysteresis loop response increased significantly with a decrease in temperature, this effect of an increase in magnetization with temperature was first observed 


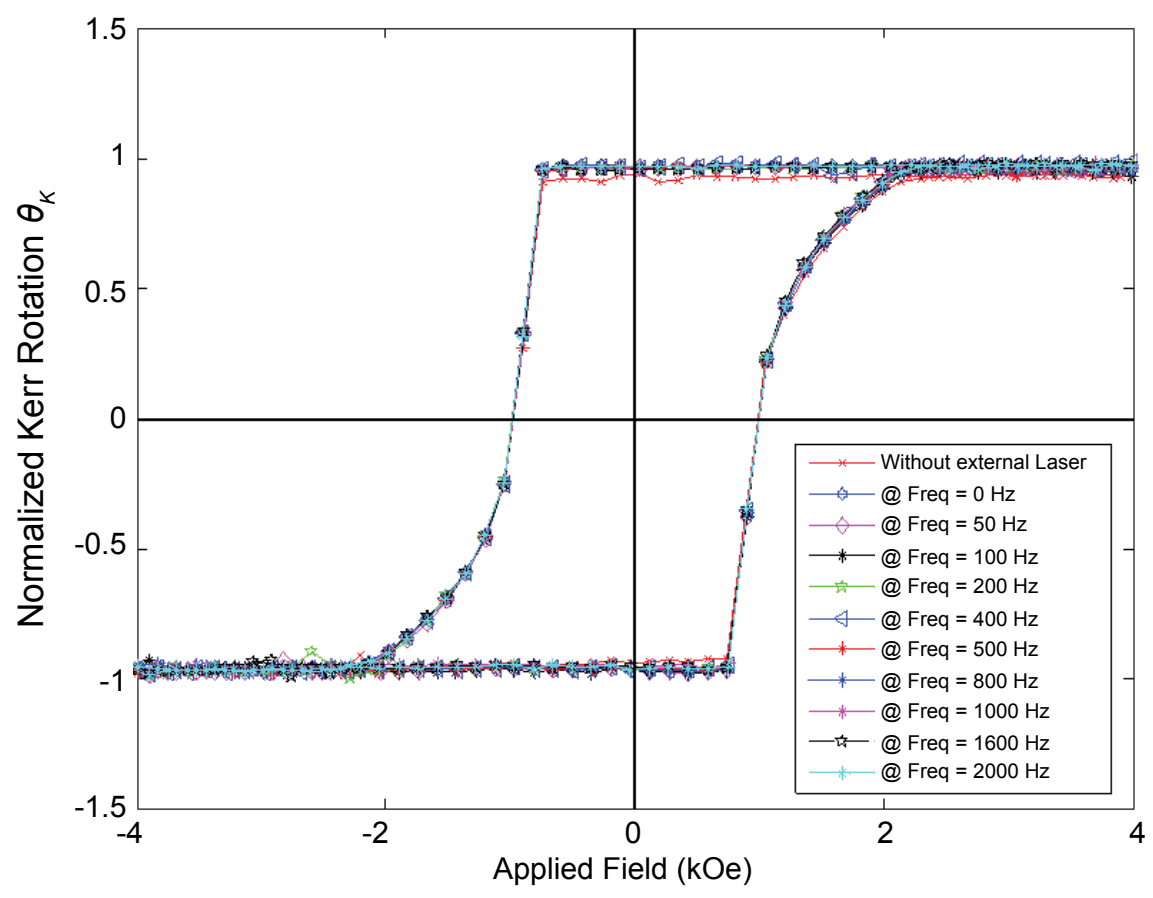

Figure 4: Kerr rotation major hysteresis loop with and without external laser modulation frequencies at room temperature.

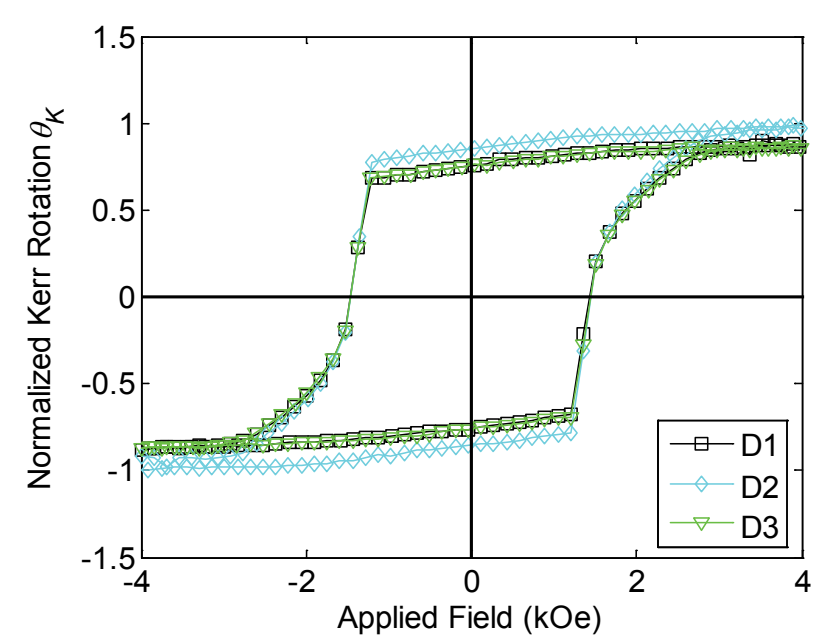

Figure 5: Kerr rotation hysteresis major loops. D1 depicts the Kerr rotation of sample S1 when laser L2 is switched on and modulated laser beam is incident on sample S2 at $60 \mathrm{~K}$. D2 depicts the Kerr rotation of sample S1 when laser L2 is switched on and modulated laser beam is incident on sample S1 at $60 \mathrm{~K}$. D3 depicts the Kerr rotation of sample $\mathrm{S} 1$ when laser $\mathrm{L} 2$ is switched at $60 \mathrm{~K}$.

by [15] and is been previously recorded [14,16]; (ii) At the coercivity field, all the curves D1-3 showed no difference between each other; (iii) At all the applied field values, curves D1 and D3 showed a negligible difference and were almost identical while, curve D2 showed an increased value from the other curves.

The measurement results with lower temperatures down to $9.5 \mathrm{~K}$ are shown in Figure 6. The curve D4 de-

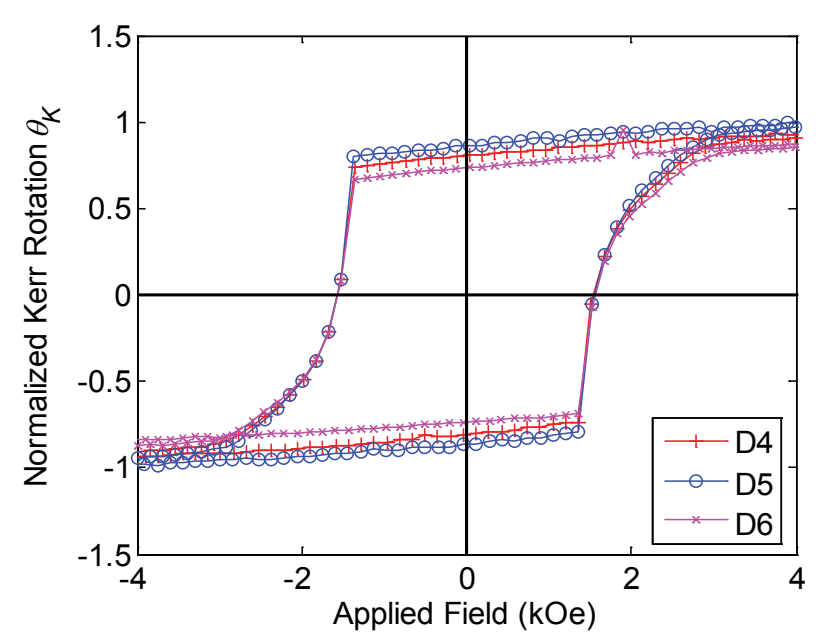

Figure 6: Kerr rotation hysteresis major loops. D4 depicts the Kerr rotation of sample S1 when laser L2 is switched on and modulated laser beam is incident on sample S2 at $9.5 \mathrm{~K}$. D5 depicts the Kerr rotation of sample S1 when laser L2 is switched on and modulated laser beam is incident on sample S1 at $9.5 \mathrm{~K}$. D6 depicts the Kerr rotation of sample $\mathrm{S} 1$ when laser $\mathrm{L} 2$ is switched off at $9.5 \mathrm{~K}$.

picts the Kerr rotation of sample S1 when laser L2 is switched on and the modulated laser beam is incident on sample S2 at $9.5 \mathrm{~K}$. The D 4 curve measures the amount of coupling from sample S2 onto sample S1. D5 depicts the Kerr rotation of sample S1 when laser L2 is switched on and the modulated laser beam is incident on sample $\mathrm{S} 1$ at $9.5 \mathrm{~K}$. The D5 curve measures the influence of the modulated laser from L2 on sample S1. D6 depicts the Kerr 


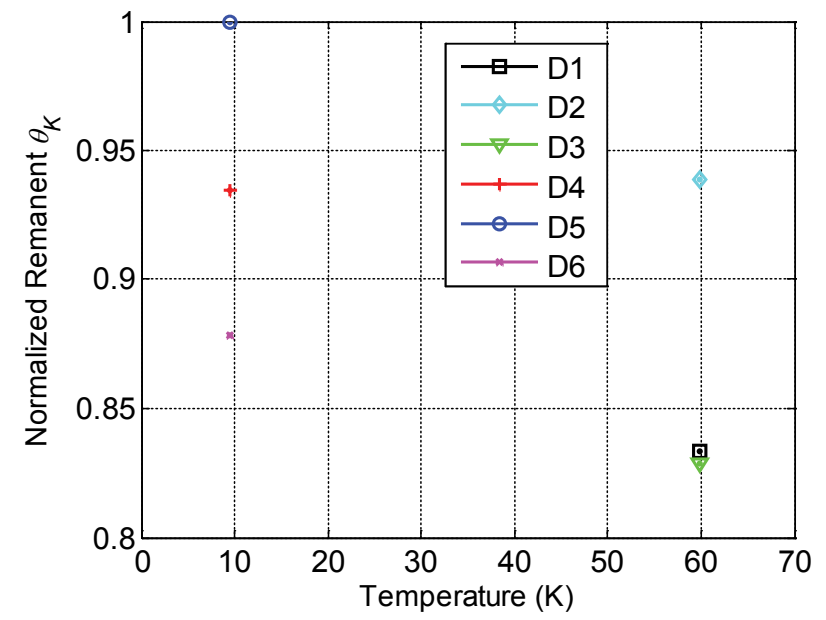

Figure 7: Normalized remanent Kerr rotation against temperature. D1 depicts the Kerr rotation of sample S1 when laser L2 is switched on and modulated laser beam is incident on sample S2 at $60 \mathrm{~K}$. D2 depicts the Kerr rotation of sample S1 when laser L2 is switched on and modulated laser beam is incident on sample S1 at $60 \mathrm{~K}$. D3 depicts the Kerr rotation of sample S1 when laser L2 is switched off at $60 \mathrm{~K}$. D4 depicts the Kerr rotation of sample S1 when laser $\mathrm{L} 2$ is switched on and modulated laser beam is incident on sample S2 at $9.5 \mathrm{~K}$. D5 depicts the Kerr rotation of sample $\mathrm{S} 1$ when laser $\mathrm{L} 2$ is switched on and modulated laser beam is incident on sample S1 at $9.5 \mathrm{~K}$. D6 depicts the Kerr rotation of sample S1 when laser L2 is switched off at $9.5 \mathrm{~K}$.

rotation of sample S1 depicts when laser L2 is switched off at $9.5 \mathrm{~K}$. This curve contains no influence of the external laser beam from L2 on sample S1. Again, we noticed the following: (i) The Kerr rotation hysteresis loop response retained its large area size with the decrease in temperature; (ii) At the coercivity field, all the curves D4-6 showed no difference between each other; (iii) At all the applied field values except at coercivity, curves D4 and D5 showed an increased value in Kerr rotation from each other and both curves had higher Kerr rotation values than D6.

The extracted remanent Kerr rotation temperature response is shown in Figure 7 which clearly shows the comparison of all the curves D1-6 with the aim to investigate the following questions:

(i) What amount of interaction will an external modulated laser beam have on the Kerr rotation of sample? This interaction arises from photon-magnon interaction in the sample.

(ii) How much of the photon-magnon interaction gets coupled onto another sample with identical material composition.

(iii) What happens when (i) and (ii) are repeated at temperatures above and below the BEC transition temperature of the sample.

The difference between curves D2 and D3 show the amount of interaction of the external modulated laser beam from L2 on the Kerr rotation of sample S1 at 60 $\mathrm{K}$ (a temperature above the BEC transition temperature for the sample S1). The difference between curves D5 and D6 show the amount of interaction of the external modulated laser beam from $\mathrm{L} 2$ on the Kerr rotation of sample $\mathrm{S} 1$ at $9.5 \mathrm{~K}$ (a temperature below the BEC transition temperature for the sample S1). Measurement curves D1 and D4 shows the amount of coupling (from the interaction of L2 laser on sample S2) gets into sample S1 at temperatures above and below the BEC transition temperature respectively.

As mentioned above the presence of the external laser beam from L2 on sample S1 creates an offset in the Kerr rotation signal and we find that this offset is analogous to the increase in the Kerr rotation loops in D2 and D5. This interesting phenomenon has not been previously reported yet. This increase is due to the photon-magnon interaction and further investigation will be conducted on the phenomenon. Furthermore, we noticed that the Kerr rotation of D5 was greater than D6, but less than D4. Thus, there is a coupling phenomenon occurring when the temperature is decreased to $9.5 \mathrm{~K}$ as opposed to when the temperature is at $60 \mathrm{~K}$. Low temperature magnetic measurements on the $\mathrm{Co} / \mathrm{Pd}$ sample with similar nanometer dimensions has been reported to have a magnon Bose-Einstein condensation (BEC) phenomenon occurring at temperatures below $35 \mathrm{~K}$ [14].

The coupling phenomenon observed is attributed to quantum entanglement between the magnons in sample S1 and S2 as explained by the following arguments:

(i) At temperatures below the BEC transition temperature of the sample, magnons confined in the sample begin to occupy the lowest energy state. Hence, at $9.5 \mathrm{~K}$, a large portion of magnons confined in both samples S1 and S2 occupy their lowest energy levels which are equal since both samples were once a single sample that was fabricated as described above in the experiment section.

(ii) The increase in the Kerr rotation does not arise from magnetic coupling between the two samples placed in closed proximity. The only coupling reported until now [17] is about the intra-layer coupling in multi-layered thin films. Also, the exchange coupling [18] is also limited to certain intra-atomic or molecular levels and not at such large distances as the two samples separated apart or the two lasers on the same sample. The magnetic field originating out of the two samples is not significant enough to be responsible for the observed coupling because at $60 \mathrm{~K}$, the samples were placed inside relatively very high field up to $4 \mathrm{kOe}$ and there was no coupling between the samples. The observed phenomenon indicates the occurrence of entanglement of magnons confined in the thin films. 


\section{Conclusion}

We have reported an experimental observation of entanglement of magnons confined in the $\mathrm{Co} / \mathrm{Pd}$ thin films at temperatures below the BEC temperature of the films. A MOKE-based system was used in the measurement. Magnon entanglement between the thin films resulted in the increase in Kerr rotation response of sample S1 via coupling from a laser modulated sample S2 within a close proximity from each other. Further research investigation of the observed magnons entanglement phenomenon will be conducted in near future. This finding paves a way for researchers to exploit quantum mechanical effects of magnons for developing novel magnetic sensors.

\section{Acknowledgement}

This research was performed at the MOKE laboratory of the Institute for Magnetics Research (IMR) at The George Washington University Virginia Campus. We would like to thank all members of the IMR group for their valuable discussion during the course of the research.

\section{References}

1. Einstein A, Podolsky B, Rosen N (1935) Can QuantumMechanical Description of Physical Reality Be Considered Complete? Physical Review 47: 777-780.

2. DL Moehring, $P$ Maunz, S Olmschenk, KC Younge, DN Matsukevich, et al. (2007) Entanglement of single-atom quantum bits at a distance. Nature 449: 68-71.

3. S Ritter, C Nölleke, C Hahn, A Reiserer, A Neuzner, et al. (2012) An elementary quantum network of single atoms in optical cavities. Nature 484: 195-200.

4. J Hofmann, M Krug, N Ortegel, L Gérard, M Weber, et al. (2012) Heralded entanglement between widely separated atoms. Science 337: 72-75.

5. Chou CW, H de Riedmatten, D Felinto, SV Polyakov, SJ van Enk, et al. (2005) Measurement-induced entanglement for excitation stored in remote atomic ensembles. Nature 438: 828-832.

6. A Stute, B Casabone, P Schindler, T Monz, PO Schmidt, et al. (2012) Tunable ion-photon entanglement in an optical cavity. Nature 485: 482-485.

7. B Weber, HP Specht, T Müller, J Bochmann, M Mücke, et al. (2009) Photon-Photon Entanglement with a Single Trapped Atom. Phys Rev Lett 102: 030501.

8. J Berezovsky, MH Mikkelsen, NG Stolz, LA Coldren, D Awschalom (2008) Picosecond coherent optical manipulation of a single electron spin in a quantum dot. Science 320: 349-352.

9. X Xu, Bo Sun, Paul R Berman, Duncan G Steel, Allan S Bracker, et al. (2008) Coherent population trapping of an electron spin in a single negatively charged quantum dot. Nat Phys 4: 692-695.

10. K De Greve, L Yu, PL McMahon, JS Pelc, CM Natarajan, et al. (2012) Quantum-dot spin-photon entanglement via frequency downconversion to telecom wavelength. Nature 491: 421-425.

11. Arnold C, Justin Demory, Vivien Loo, Aristide Lemaître, Isabelle Sagnes, et al. (2015) Macroscopic rotation of photon polarization induced by a single spin. Nat Commun 6: 6236.

12. CA Nwokoye, LH Bennett, E Della Torre, A Siddique, FA Narducci, et al. (2015) Low Temperature Magneto-Optical Kerr Effect Experimental System with a Cryogen-Free Sample Environment. International Journal of Nanoparticles and Nanotechnology 1: 1-6.

13. T Morimae, A Sugita, A Shimizu (2005) Macroscopic entanglement of many-magnon states. Physical Review A 71: 032317.

14. CA Nwokoye, LH Bennett, E Della Torre, M Ghahremani, FA Narducci (2017) Magneto-optical and magnetic properties in a Co/Pd multilayered thin film. Journal of Magnetism and Magnetic Materials 421: 230-233.

15. F Bloch (1930) Zur Theorie des Ferromagnetismus. Zeitschrift für Physik 61: 206-219.

16. Della Torre E, LH Bennett, RE Watson (2005) Extension of the Bloch T 3/2 law to magnetic nanostructures: Bose-Einstein condensation. Phys Rev Lett 94: 147210.

17. J Bruyere, O Massenet, R Montmory, L Neel (1965) A coupling phenomenon between the magnetization of two ferromagnetic thin films separated by a thin metallic film--Application to magnetic memories. IEEE Transactions on Magnetics 1: 10-12.

18. Bell JS (1964) On the Einstein Podolsky Rosen Paradox. Physics 1: 195-200. 